\title{
Application of sub-THz Circular Dichroism Quasi-Optics to Probe the Conformality of Solvated Protein
}

\author{
Jhih-Hong Cheng ${ }^{1}$, Robert C. Jones ${ }^{1}$, Oleksandr Sushko ${ }^{2}$, Bin Yang ${ }^{3}$, Yumiko Tashiro ${ }^{1}$, and Robert Donnan ${ }^{1}$ \\ ${ }^{1}$ Queen Mary, University of London, London, UK \\ ${ }^{2}$ Igor Sikorsky Kyiv Polytechnic institute, Kyiv, Ukraine \\ ${ }^{3}$ University of Chester, Chester, UK
}

\begin{abstract}
A sub-terahertz (sub-THz) Circular Dichroism (CD) quasi-optical (QO) system has shown utility in acquiring electromagnetic absorption signatures of solvated protein samples over $\mathrm{H}$-band frequencies $(220-325 \mathrm{GHz})$. We report the sub- $\mathrm{THz}$ CD signature of Myoglobin in particular. Spectra are reproducible within experimental error.
\end{abstract}

\section{INTRODUCTION}

$\mathrm{s}$ ince the 2000s, sub-THz and THz signals and circuits have seen increasing application in the study of manifold phenomena in molecular biological science; the complexity of the folding process of proteins being among the most challenging. This is because such frequencies are the natural probe for sensing the characteristic vibrational modes that drive and facilitate such processes. But attendant to the necessity of an aqueous environment to ensure a meaningful study of protein folding [1], is the inescapable frustration that this very same water exacts a heavy attenuation on the probe signal; $180 \mathrm{~dB} . \mathrm{mm}^{-1}$ ! [2]. Hence, early THz spectroscopy, such as $\mathrm{THz}$ time-domain spectroscopy (TDS), worked with protein samples that were mostly of a dry, powdered form [3]. A protein must be hydrated to at least $30 \%$ of its total weight in order for its 'backbone' to be well-defined [4]. Water forms a hydrogenbond network that envelopes and couples to the protein, promoting the drive to fold the protein to its stable, preprogramed conformational state. All biological polymers, such as amino acids, peptides, proteins and higher-level structures such as DNA and RNA, when folded, exhibit chirality (lefthanded predominating); these molecules possessing chiral chromophores. CD spectroscopy is therefore a natural tool to observe this; additionally so, since water of itself is achiral, and so fails to confound the acquired CD spectrum. CD is given to be the difference in absorption between left- and right-handed circularly-polarized light. $\mathrm{THz} \mathrm{CD}$, calibrated to known conformational behavior measured by standard far-UV CD, is explored here as a complementary spectroscopy to probe the conformation of dissolved protein. It is observed that $\mathrm{THz} \mathrm{CD}$ is more sensitive to secondary and tertiary conformation states, suggesting its utility for fine-structure discrimination and tracking in response to variation in environmental factors such as $\mathrm{pH}$, temperature and solute concentration.

\section{Method 1 And Method 2 Measurement}

An Agilent Technologies Vector Network Analyzer (N5244A VNA)-driven QO $\mathrm{THz}$ CD spectrometer was configured as shown in Fig. 1. The VNA has a baseband spanning $10 \mathrm{MHz}$ to $43.5 \mathrm{GHz}$. Higher-frequency waveguide bands are accessed by attachment of frequency-extender modules, to which are attached appropriate matched-pairs of
ultra-Gaussian, high-gain, corrugated, profiled, transmit $\left(\mathrm{T}_{\mathrm{x}}\right)$ and receive $\left(\mathrm{R}_{\mathrm{x}}\right)$ horns $\left(\mathrm{H}_{1}\right.$ and $\mathrm{H}_{2}$ in Fig. 2$)$. The system allows for coverage up to $1.1 \mathrm{THz}$.

(a)

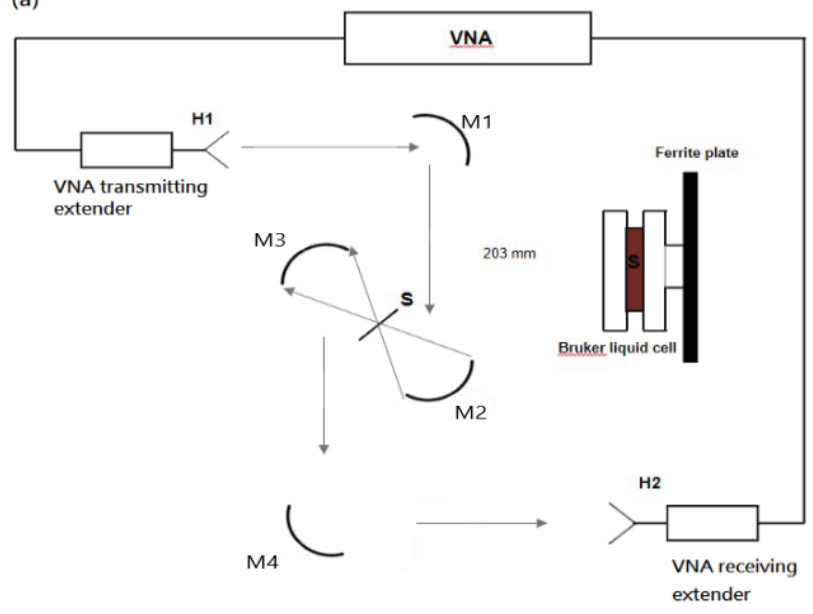

(b)

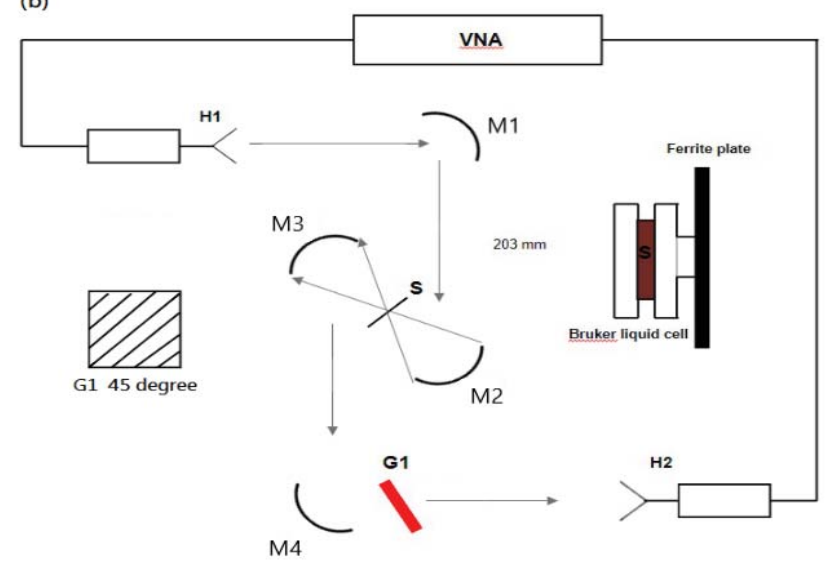

Figure 1: Schematic of the sub-THz quasi-optical apparatus and circuit for implementing circular dichroism spectroscopy. (a) Method 1 measurement and (b) Method 2 measurement. A $\mathbf{4 5 ^ { \circ }}$ wire-grid polarizer G1 interleaves a fast parabolic mirror, $\mathrm{M} 4$, and receive-horn, $\mathrm{H} 2$, to measure a $\mathbf{4 5 ^ { \circ }}$ polarized signal to obtain post-processing derivation of cross-polar radiation. Method 2 obviates the need to physicaly rotate the horn and consequently move cabling, which would consequently corrupt the initial phase-callibration.

The off-axis ellipsoidal mirror (M1) is set to efficiently receive the beam-field from $\mathrm{T}_{\mathrm{x}}(\mathrm{H} 1)$ and couple to a fast, parabolic mirror, M2, which is one in the confocal pair with M3, at whose common focus the sample (S) is situated. M1 and M4, have a $250 \mathrm{~mm}$ focal length and M2 and M3 a focal length of $100 \mathrm{~mm}$.

A Bruker liquid cell was used as a sample holder. It consists of oppositely facing neoprene gaskets, TPX plates separated by 
a PTFE spacer. The spacer imposes a gap of $100 \mu \mathrm{m}$ (fixing the effective one-way beam-path length through the solution). A ferrite plate was induced prior to the sample. It introduces a Faraday rotation upon the vertical, linearly-polarized signal beam from $\mathrm{T}_{\mathrm{x}}$ and serves to amplify the cross-polar transmission, which otherwise is inherently week (for purposes of CD spectroscopy). Co- and cross-polarised signal components are use in post-processing to generate the left- and right-handed senses of circular polarisation required for a $\mathrm{CD}$ measurement [5]. In CD, RHCP typically dominates over LHCP. In order, then, to have a workable CD signature, LHCP has this effective gain applied to it as a simple scaling factor. $C D$ is unique in spectroscopy in that the feature of interest is the frequency at which the trace crosses from positive to negative values (i.e. the 'zero-crossing'), as distinct from where peaks (or troughs) are centred, as in conventional spectroscopy.

The traditional measurement methodology, Method I, is used to measure the inherent propagation of circularly polarised transmission through the sample by measurement of vertical (co-polar) and horizontal (cross-polar) transmittances. So horn $\mathrm{H} 2$ and extender, are physically rotated through $90^{\circ}$ for acquiring the cross-polar signal component, as in the Fig.1 (a). The expressions for right- and left- handed circularly polarized waves are, respectively $[5,6]$ :

$$
\begin{aligned}
& T_{+}(\omega) \equiv \frac{1}{\sqrt{2}}(\operatorname{Tcr}(\omega)-i \operatorname{Tco}(\omega)) \text { and } \\
& T_{-}(\omega) \equiv \frac{1}{\sqrt{2}}(\operatorname{Tcr}(\omega)+i \operatorname{Tco}(\omega)) .
\end{aligned}
$$

Using $T_{+}(\omega)$ and $T_{-}(\omega)$, RHCP and LHCP for generating the $\mathrm{CD}$ spectrum $\Delta \mathrm{T}(\omega)$ work together as $[5,6]$ :

$$
\Delta \mathrm{T}(\omega)=\left|T_{+}(\omega)\right|-\left|T_{-}(\omega)\right| .
$$

Method 1 of physically rotating $\mathrm{R}_{\mathrm{x}}$ and its frequencyextender module unavoidably involved cable-movement that corrupts earlier calibration and introduces a significant source of systematic error. To avoid this, Method 2 was innovated with the introduction of a $45^{\circ}$ wire-grid polarizer interleaving mirror $\mathrm{F} 4$ and $\mathrm{R}_{\mathrm{x}}$ horn, $\mathrm{H} 2$, to obtain an equivalent cross-polar signal (Fig. 1(b)). According to Jones' calculus, the linearly-polarized signal at $45^{\circ}$ to the $\mathrm{x}$-axis, $T_{45}$, can be calculated by horizontal and vertical components as [6]

$$
T_{45}(\omega)=\frac{1}{\sqrt{2}}(\operatorname{Tcr}(\omega)+\operatorname{Tco}(\omega)) .
$$

To calculate the cross-polar signal, we can rewrite Eq. 4 as below [6]

$$
\operatorname{Tcr}(\omega)=\sqrt{2} T_{45}(\omega)-\operatorname{Tco}(\omega) .
$$

The advantage of this procedure is that the determination of cross-polar preserves initial calibration.

\section{RESULT}

Fig. 2 shows the comparison of Methods 1 and 2 by CD characterization of a dissolved Myoglobin liquid (with $\mathrm{pH} 7$ at $5 \mathrm{mg} \cdot \mathrm{ml}^{-1}$ ). It shows the analysis of Method 2. Thirteen 'zerocrossings' are observed. Their positioning is characteristic to the sample and acts as its 'finger print'. The frequency shift of zero-crossings is caused by rotational and vibrational modes of the secondary structure of the protein and surrounding molecules. The red line in Fig. 3 represents the free-space CD
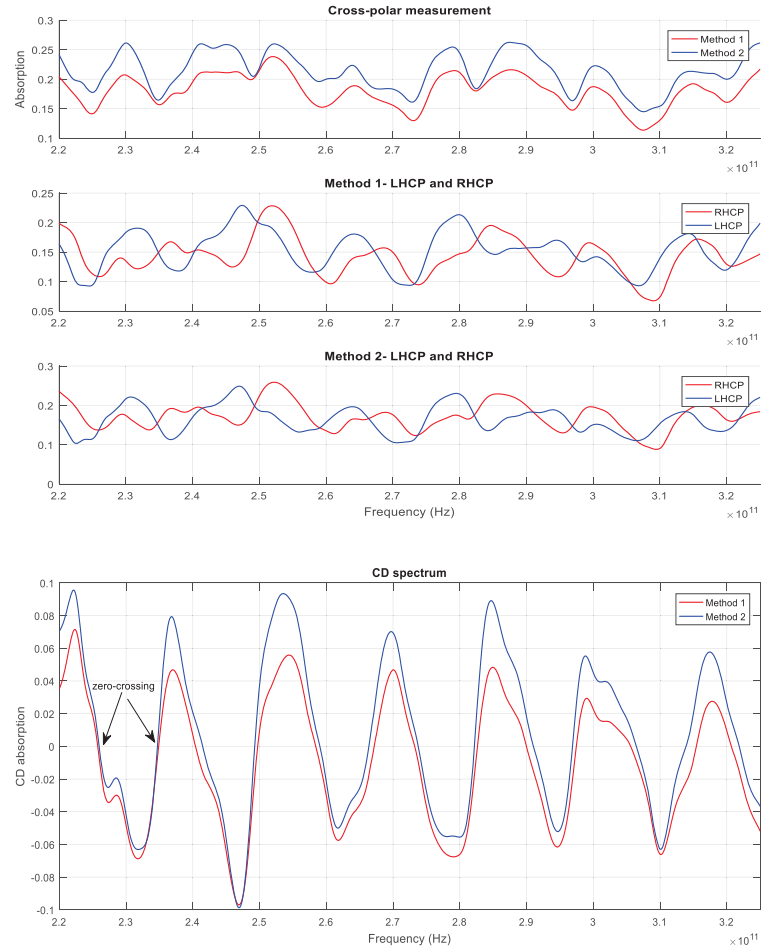

Figure 2: Comparison of Methods 1 and 2: The upper plot shows the measured versus derived cross-polar signal. The second and the third plots show the rightand left-hand behaviour of circularly polarized waves according to Methods 1 and 2. The last plot shows the comparisons of CD spectra.

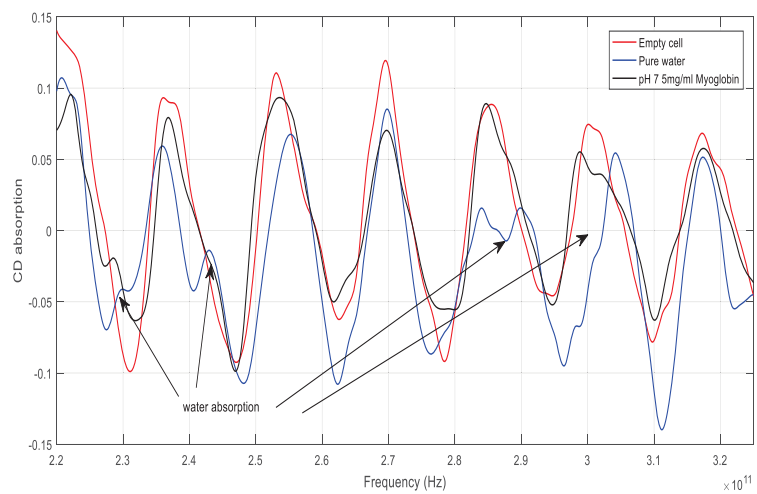

Figure 3: $\Delta \mathbf{T}(\boldsymbol{\omega})$ of an empty cell (red), and pH $75 \mathrm{mg} / \mathrm{ml}$ Myoglobin (black). Since pure water (blue) is achiral and suffer strong water absorption, water does not have $\mathrm{CD}$ response.

spectrum for the empty sample-cell. Subsequent samples have their distinctive absorptive action on propagating circularly polarized signals that ultimately leads to characteristic shifts of these zero-crossing points. By injecting de-ionised water into the empty cell, the CD spectrum suffers water absorption at 230, 243, 288 and $300 \mathrm{GHz}$. However, by dissolving Myoglobin in a buffer solution, water molecules are displaced and the effect is seen by the black absorption trace of Fig. 3. Although strong water absorption at 288 and $300 \mathrm{GHz}$ exhibits extra zerocrossings and is the cause of a series of frequency shift, the CD spectra clearly demonstrate discriminative sensitivity. The number of zero-crossing points can, however, also be reduced by increasing the optical path-length. The depth of sample is set to $100 \mu \mathrm{m}$ as a trade-off between beam-sample interaction and $\mathrm{S} / \mathrm{N}$. 


\section{Conclusion}

A sub-THz Circular Dichroism Quasi-Optical spectrometer operating from 220 to $325 \mathrm{GHz}$ is described. An innovation (Method 2) is outlined that clarifies application of circular dichroism at mm-wavelengths for observing secondary and tertiary conformations of dissolved myoglobin. Nonetheless the path-length for beam-sample interaction needs careful consideration to ensure a workable dynamic range to mitigate against signal loss due to water absorption.

\section{REFERENCES}

[1] M. Woerner, W. Kuehn, P. Bowlan, K. Reimann and T. Elsaesser, "Ultrafast two-dimensional terahertz spectroscopy of elementary excitations in solids", New Journal of Physics, vol. 15 , no. 2, p. $025039,2013$.

[2] Freeman, T. (2018). THz technology: a new take on cancer imaging - Physics World. [online] Physics World. Available at: https://physicsworld.com/a/thz-technology-a-new-takeon-cancer-imaging/

[3] O. Sushko, R. Dubrovka and R. Donnan, "Terahertz Spectral Domain Computational Analysis of Hydration Shell of Proteins with Increasingly Complex Tertiary Structure", The Journal of Physical Chemistry B, vol. 117, no. 51, pp. 1648616492, 2013.

[4] R. Singh, D. George, C. Bae, K. Niessen and A. Markelz, "Modulated orientation-sensitive terahertz spectroscopy", Photonics Research, vol. 4, no. 3, p. A1, 2016

[5] B. Yang, R. Wylde, D. Martin, P. Goy, R. Donnan and S. Caroopen, "Determination of the Gyrotropic Characteristics of Hexaferrite Ceramics From 75 to $600 \mathrm{GHz}$, IEEE Transactions on Microwave Theory and Techniques, vol. 58, no. 12, pp. 3587-3597, 2010.

[6] R. Lewis, Terahertz physics. Cambridge: Cambridge University Press, 2012. 